\title{
Determination of malondialdehyde, Uric Acid, Bilirubin And Total Antioxidant Status, in Children Under 5 Years Suffering From Malaria in Osogbo, Nigeria
}

\author{
M.J.Olisekodiaka ${ }^{1}$,A.J.Onuegbu ${ }^{1}$,C.Igbeneghu ${ }^{2}$, N.Nwagbo $^{2}$, U.K.Amah ${ }^{1}$, \\ And J.E.Okwara ${ }^{1}$ \\ ${ }^{I}$ Department Of Chemical Pathology, Faculty Of Medicine, Nnamdi Azikiwe University, Awka, Nigeria \\ ${ }^{2}$ Department Of Biomedical Sciences, College Of Health Sciences, Ladoke Akintola University Of Technology, \\ Ogbomoso, Nigeria
}

\begin{abstract}
Background: Children are prone to malaria infection. Peroxidation of membranes lipids, hemolysis and oxidative stress due to invasion of erythrocytes by malaria parasites could worsen the disease and increase mortality. Aim: Evaluation of Malondialdehyde (MDA), Total Antioxidant Status (TAS), uric acid and bilirubin in children suffering from malaria.

Materials and Method: Thirty malaria patients (age 1-5 years) were divided into two groups based on density of parasitaemia: Group1 $=$ thirteen children scored as $(+)$ and group $2=$ seventeen children scored as $(++)$. Thirty apparently healthy children (age 1-5 years, parasitaemia score = -) served as control. Serum from groups 1, 2 and control were used to determine levels of uric acid, TAS, MDA and bilirubin by standard spectrophotometric methods. Results: Results showed that mean uric acid in groupl $(5.28 \pm 0.19)$ and group 2 $(5.96 \pm 0.25)$ as well as MDA levels (group $1=6.36 \pm 0.17$; group $2=7.97 \pm 0.44)$ were higher than means of corresponding control (uric acid $=3.69 \pm 0.10, M D A=1.02 \pm 0.06$ ) respectively. Lower TAS level (group $1=1.16$ \pm 0.02 group $2=0.92 \pm 0.06)$ than control $(1.44 \pm 0.01)$ was observed. Conclusion:Malarialeads to increased peroxidation, haemolysis and oxidative stress possibly resulting in depletion of the body's antioxidant defenses and decreased TAS. Children, generally not enthusiastic about intake of fruits and vegetables may benefit from antioxidant supplementation during malaria bouts.
\end{abstract}

Keywords: Malaria, Haemolysis, Oxidative Stress, Lipid Peroxidation, Antioxidants

\section{Introduction}

Malaria is a mosquito-borne infectious disease of humans caused by eukaryotic protists of the genus Plasmodium are responsible for more than 500 million clinical cases of malaria globally each year ${ }^{[1]}$. About 1.5 -3.5 million deaths from malaria have been reported to occur annually and of these deaths the overwhelming majority is among children aged $<5$ years old Several studies showed that physicochemical changes in the membrane of the erythrocyte induced by oxidative stress is responsible for membrane lipid peroxidation and increased haemolysis seen in malaria patients ${ }^{[3,4,5]}$. Orengo and colleaguesshowed that after haemolysis, uric acid derived from hypoxanthine accumulated by Plasmodium falciparum-infected erythrocytes is a major contributor to the inflammatory response triggered in human peripheral blood mononuclear cells (PBMCs) ${ }^{[6]}$. The immune system of the body is activated by infections, including malaria, thereby causing the release of reactive oxygen species. The accumulation of organic peroxides and oxidation of membrane lipids place a stress on cellular vitality ultimately leading to destructive effects on the cell. The production of malondialdehyde is used as a biomarker to measure the level of oxidative stress in an organism ${ }^{[7]}$.

Antioxidants are substances which when present in low concentration compared to the oxidizable substrate, significantly delay or inhibit the oxidation of that substrate ${ }^{[8]}$. The physiological role of antioxidants is to prevent damage to cellular components arising from the activity of chemical reactions involving free radicals seen in oxidative stress. There are three classes of antioxidants namely: primary antioxidants ${ }^{[9]}$, secondary antioxidants ${ }^{[10,11]}$ and tertiary antioxidants ${ }^{[12]}$. All these antioxidants in the body together form the total antioxidant status (TAS) of an individual.Bilirubin is the yellow breakdown product of normal hemecatabolism after haemolysis. Heme is found in hemoglobin, a principal component of red blood cells. So far few studies have been carried out on the extent of oxidative stress, lipid peroxidation and haemolysis in malarious children living in Southwestern Nigeria. Therefore this study is designed to determine possibleassociations and relationships betweenmalaria infection and oxidative stress by assessing changes in uric acid, total antioxidant status (TAS), malondialdehyde (MDA) and bilirubin in children under 5 years of age in Osogbo, Osun state, Southwest Nigeria. 


\subsection{Subject selection}

\section{Materials}

The study involved thirty (30) malariapatients (age range 1-5 years) diagnosed on the basis of clinical findings and positive peripheral blood smear for malaria parasite and thirty subjects, who were found to be negative for P.falciparum in their peripheral blood served ascontrol. These subjects were children that attended Osun State University Teaching Hospital (OSUTH), Asubiaro, Osogbo and Maternity Center Olorunsogo, Egbedore Local Government Area, Osogbo, Osun State, Nigeria. The malaria patients were further subdivided into two groups on the basis of the presence and density of P. falciparum in each blood sample (group $1=+$ and group $2=++$ )

\subsection{Collection and storage of Blood Samples}

$5 \mathrm{ml}$ of venous blood was obtained from each malaria patient and control subjects by venopuncture before they were administered with any antimalaria therapy at the time of hospitalization.Blood was allowedto clot at room temperature and serum was obtained by centrifugation at 3000rpm for 5 minutes. Serum total and conjugated bilirubin concentrations were determined immediately while the remaining serumwas stored at -20 ${ }^{0} \mathrm{C}$ until used for the measurements of uric acid, MDA and TAS.

\subsection{Parasitology}

The presence and density of P. falciparum in each blood sample was determined from Giemsa-stained thick blood films. A slide was scored as negative if 100 high power fields (at 100x objective) had been examined carefully without seeing any parasites. The amount of parasites in positive smears was counted to determine the intensity of infection. Positive smears were grouped into 2 (high field power 1-10= MP+, high field power $11-100=\mathrm{MP}++$ )based on the criteria described byCheesbrough ${ }^{[13]}$

\section{Methods}

\subsection{Methods of Assay of uric acid, total antioxidant status, malondialdehyde and bilirubin}

Assay for serum uric acid was carried out according to the protocol described by Fossati and coworkers $^{[14]}$,Malondialdehyde (MDA) level was estimated according to the protocol outlined by Gutteridge and Wilkins ${ }^{[15]}$ andtotal antioxidant status was measured according to the spectrophotometric method of Koracevic and colleagues ${ }^{[16]}$. Serum bilirubin was assayed by the modified method of Malloy and Evelyn ${ }^{[17]}$. Resultsobtained for MDA was calculatedas described by Buege and Aust ${ }^{[18]}$.

\subsection{Statistical Analysis}

Results obtained from this study were reported as mean $( \pm \mathrm{SE})$. Means of the parameters were analyzed using the analysis of variance (ANOVA) test and pairwise t-test was used to compare significant variables. Pearson's correlation coefficient (r) was used to determine the relationship between means of the parameters in the groups. Results were regarded as significant at $\mathrm{p}<0.05$.

\section{Results}

Results from the present study showed that the means of MDA, TAS, uric acid and bilirubin were higher in the test group when compared with the corresponding control $(\mathrm{p}<0.001)$ (Table 1)

When the test group was categorized into two groups based on the density of the malaria parasite (MP+ and MP++), mean MDA, TAS, uric acid and bilirubin were significantly higher than respective means of the corresponding control group (table 2). However, mean TAS in MP++ was higher than the value of TAS in the control group (Table 3). When means of MDA, TAS, uric acid and bilirubin of MP+ were compared with respective means of $\mathrm{MP++}$, a significantly higher MDA and uric acid values were observed in MP++ group while a lower Mean TAS was seen in MP++ group when the mean value was compared with the corresponding value in MP+ group (Table 4). Negative Pearson's correlation coefficient (r) was observed between uric acid and TAS $(r=-0.567, \mathrm{p}<0.05)$ and between total bilirubin and TAS $(r=-0.716, p<0.01)($ Table 5)

Table 1 Means (SEM), p-values of biochemical parameters in test and control groups

\begin{tabular}{|l|l|l|l|l|}
\hline Parameters & \multicolumn{1}{|c|}{ Test $(\mathrm{n}=30)$} & \multicolumn{1}{|c|}{ Control $(\mathrm{n}=30)$} & t-value & p-value \\
\hline MDA $\mu \mathrm{mol} / 1$ & $7.05 \pm 0.26$ & $1.02 \pm 0.06$ & 22.863 & 0.001 \\
\hline TAS $\mu \mathrm{mol} / \mathrm{l}$ & $1.05 \pm 0.04$ & $1.44 \pm 0.01$ & -10.211 & 0.001 \\
\hline Uric acid mg/dl & $5.58 \pm 0.16$ & $3.69 \pm 0.10$ & 9.887 & 0.001 \\
\hline TB $\mu \mathrm{mol} / 1$ & $20.77 \pm 0.26$ & $12.27 \pm 0.37$ & 18.727 & 0.001 \\
\hline $\mathrm{CB} \mu \mathrm{mol} / \mathrm{l}$ & $7.13 \pm 0.22$ & $3.67 \pm 0.19$ & 11.876 & 0.001 \\
\hline
\end{tabular}

$\mathrm{TAS}=$ total antioxidant status, $\mathrm{MDA}=$ Malondialdehyde, $\mathrm{TB}=$ total bilirubin, $\mathrm{CB}=$ conjugated bilirubin 
Table 2. Means (SEM), p-values of biochemical parameters in test group I (MP +) and control

\begin{tabular}{|l|l|l|l|l|}
\hline Parameters & MP $(+), \mathrm{n}=13$ & \multicolumn{1}{|c|}{ Control,n=30 } & t-value & -value \\
\hline MDA $\mu \mathrm{mol} / 1$ & $6.36 \pm 0.17$ & $1.02 \pm 0.06$ & -29.344 & 0.001 \\
\hline TAS $\mu \mathrm{mol} / 1$ & $1.16 \pm 0.02$ & $1.44 \pm 0.01$ & 13.309 & 0.001 \\
\hline Uric acid mg/dl & $5.28 \pm 0.19$ & $3.69 \pm 0.10$ & -8.223 & 0.001 \\
\hline TB $\mu \mathrm{mol} / 1$ & $20.47 \pm 0.35$ & $12.27 \pm 0.37$ & -14.510 & 0.001 \\
\hline $\mathrm{CB} \mu \mathrm{mol} / 1$ & $7.00 \pm 0.32$ & $3.67 \pm 0.19$ & -9.617 & 0.001 \\
\hline
\end{tabular}

$\mathrm{TAS}=$ total antioxidant status, $\mathrm{MDA}=$ Malondialdehyde, $\mathrm{TB}=$ total bilirubin, $\mathrm{CB}=$ conjugated bilirubin As shown in table 2, when the test group was divided based on parasite density,

Table 3. Means (SEM), p-values of biochemical parameters in test group I $(\mathrm{MP}++)$ and control

\begin{tabular}{|l|l|l|l|l|}
\hline Parameters & MP $(++), n=17$ & Control,n=30 & t-value & p-value \\
\hline MDA $\mu \mathrm{mol} / 1$ & $6.36 \pm 0.17$ & $1.02 \pm 0.06$ & -15.652 & 0.001 \\
\hline TAS $\mu \mathrm{mol} / 1$ & $1.16 \pm 0.02$ & $1.44 \pm 0.01$ & 8.293 & 0.001 \\
\hline Uric acid mg/dl & $5.28 \pm 0.19$ & $3.69 \pm 0.10$ & -8.339 & 0.001 \\
\hline TB $\mu \mathrm{mol} / 1$ & $20.47 \pm 0.35$ & $12.27 \pm 0.37$ & -14.404 & 0.001 \\
\hline CB $\mu \mathrm{mol} / 1$ & $7.00 \pm 0.32$ & $3.67 \pm 0.19$ & -10.416 & 0.001 \\
\hline
\end{tabular}

$\mathrm{TAS}=$ total antioxidant status, $\mathrm{MDA}=$ Malondialdehyde, $\mathrm{TB}=$ total bilirubin, $\mathrm{CB}=$ conjugated bilirubin

Table 4. Means (SEM), p-values of biochemical parameters in test group based on density of malaria parasites

\begin{tabular}{|l|l|l|l|l|}
\hline Parameters & MP $(+) \mathrm{n}=13$ & MP $(++) \mathrm{n}=17$ & $\mathrm{t}$-Value & p-Value \\
\hline MDA $\mu \mathrm{mol} / 1$ & $6.36 \pm 0.17$ & $7.97 \pm 0.44$ & -3.419 & 0.004 \\
\hline TAS $\mu \mathrm{mol} / 1$ & $1.16 \pm 0.02$ & $0.92 \pm 0.06$ & 3.772 & 0.002 \\
\hline Uric Acid mg/dl & $5.28 \pm 0.19$ & $5.96 \pm 0.25$ & -2.218 & 0.035 \\
\hline TB $\mu \mathrm{mol} / 1$ & $20.47 \pm 0.35$ & $21.15 \pm 0.36$ & -1.336 & 0.192 \\
\hline CB $\mu \mathrm{mol} / 1$ & $7.00 \pm 0.32$ & $7.31 \pm 0.31$ & -0.676 & 0.505 \\
\hline
\end{tabular}

$\mathrm{TAS}=$ total antioxidant status, $\mathrm{MDA}=$ Malondialdehyde, $\mathrm{TB}=$ total bilirubin, $\mathrm{CB}=$ conjugated bilirubin

Table 5. Pearson's Correlation coefficient (r) of biochemical parameters in MP(+) and MP (++)groups

\begin{tabular}{|l|l|l|l|}
\hline Parameters & Parasite density group & Pearson's correlation coefficient (r) & p-value \\
\hline Uric acid and TAS & + & -0.567 & $<0.05$ \\
\hline TB and TAS & ++ & -0.716 & $<0.01$ \\
\hline TB and CB & ++ & 0.614 & $<0.01$ \\
\hline TB and uric acid & ++ & 0.719 & $<0.01$ \\
\hline TB and MDA & ++ & 0.683 & $<0.05$ \\
\hline & & & \\
\hline
\end{tabular}

$\mathrm{TAS}=$ total antioxidant status, $\mathrm{MDA}=$ Malondialdehyde, $\mathrm{TB}=$ total bilirubin, $\mathrm{CB}=$ conjugated bilirubin

\section{Discussion}

Asignificantly higher uric acid level in malarious children seenin this study is similar with the reports from previous studies ${ }^{[19,20,21]}$ which showed an elevated level of uric acid in the blood of malaria infected humans and mice.A suggested mechanism for this observation was the presence of hypoxanthine and xanthine in both erythrocytes and the Plasmodium parasite. Hypoxanthine and xanthine is metabolized by the activities of the enzyme xanthine oxidase but the activity of xanthine oxidase has not been detected in erythrocytes or in the Plasmodium parasite. It is possible that the Plasmodium parasite imports excessive hypoxanthine and xanthine into the infected erythrocytes ${ }^{[22]}$. Since the enzyme is absent, imported hypoxanthine/xanthine would not be degraded into uric acid in erythrocytes. However, upon haemolysis, the accumulated hypoxanthine/xanthine would be released into the extracellular medium where they are degraded by xanthine oxidase to form uric acid. This process also results in the production of reactive oxygen species ${ }^{[23]}$.In humans, uric acid is the final product of hypoxanthine degradation because uricase enzyme was mutated throughout evolution and its activity cannot be found in human serum ${ }^{[24]}$. High concentrations of soluble uric acid induces the release of inflammatory mediators from different cell types including immune cells ${ }^{[25,26,27]}$ suggesting that the soluble uric acid formed via hypoxanthine degradation could also contribute to the malaria induced inflammatory response.

Total antioxidant status (TAS) level observed in children with malaria infection was significantly lower than the level for healthy children. A previous study by Akpotuzor and colleagues[28], also reported a significantly reduced level of TAS in malaria infected children when compared to healthy childrenin Calabar, South -South Nigeria. The lower value observed in TAS in malaria may be attributed to increased utilization of the host's plasma antioxidants by the malaria parasites to counteract oxidative damages.Additionally,increasedconsumption and degradation of antioxidants and antioxidant enzymes as well as haemoglobin by malaria parasite to produce its own proteins has been reported by Koracevic and coworkers $^{[16]}$.

The significant increase in malondialdehyde (MDA) concentration observed in malarious childrenseen in this present study might reflect the extent of lipid peroxidation and is supported by previous reports ${ }^{[29,30]}$ 
where the authors reported an increased MDA concentration in malarious children both in Eku metropolis and Benin City, both in Mid-Western Nigeria.Some other studies ${ }^{[31,32]}$ also showed increased lipid peroxidation in malaria. Invasion of human erythrocytes by malaria parasite results in vulnerability to damage of the erythrocytes due to toxic metabolites derived from both the host cells and parasites. Reactive oxygen species (ROS) generated in the host-parasite interaction which could lead to the lysis of erythrocytes and possible alterations in the antioxidant defense system ${ }^{[33]}$.

Increased bilirubin level was observed in malarious children compared with healthy children in this study. A similar observation was reportedin the study of Adeosun and colleagues ${ }^{[34]}$ in Ile-Ife, South Western Nigeria which showed thathyperbilirubinemia occurred in malarious children compared with the controls.In another study, authors noted that the possible causes of hyperbilirubinemia were multi-factorial and include intravascular haemolysis of parasitized RBCs as well as haemolysis of non-parasitized $\mathrm{RBCs}^{[35]}$.

Increased uric acid and bilirubin concentrations probably indicate increased haemolysis arising as a result of increased lipid per oxidative damage to the red blood cells. An indication for increased lipid peroxidation was given by the significant increase in MDA level seen in the children suffering from malaria.

\section{Conclusion}

In conclusion, Increased MDA, uric acid and bilirubin in malarious children may be attributed to the increased peroxidation, haemolysis and oxidative stress resulting in depletion of the body's antioxidant defenses and decreased TAS. Children, generally not enthusiastic about intake of fruits and vegetables may benefit from antioxidant supplementation during malaria bouts.

\section{References}

[1]. R.W Snow, C.A Guerra, A.M Noor, H.Y. Myint, and S.I. Hay,The global distribution of clinical episodes of Plasmodium falciparum malaria. Nature. 4342005: 214-217

[2]. P. Trigg and A.V. Kondrachin, The current global malaria situation. Malaria, $4^{\text {th }}$ edition, London, Butterworth Publication (1993) $(11-21)$

[3]. I.A. Clark and N.H. Hunt, Evidence of reactive oxygen intermediates causing haemolysis and parasite death in malaria. Infection and Immunity. 39, 1983: $1-6$.

[4]. I.A. Clark, N.H. Hunt, W.B Cowden, L.E. MaxwellE.J. and Mackie, Radical-mediated damage to parasites and erythrocytes in Plasmoduim vinckei infected mice after injection of t-butyl hydroxide. Clinical Experimental Immunol. 56,1984: 524-530

[5]. B.S. Das and N.K. Nanada, Evidence for erythrocyte lipid peroxidation in acute falciparum malaria. Trans. Roy. Soc. Trop. Med. Hyg. 93, 1999: 58-62

[6]. J.M. Orengo, A. Leliwa-Sytek, J. E Evans, B. Evans, D. van de Hoef, M. Nyako, K. Day and A. Rodriguez, Uric acid is a mediator of the Plasmodium falciparum-induced inflammatory response. PLoS ONE. 4 (4) 2009: e5194

[7]. K. Moore and L. J. Robert, Measurement of lipid peroxidation. Free Radical Res. 28, 1998:659-671

[8]. Halliwell, B. and Gutteridge, J.M.C. (). The definition and measurement of antioxidants in biological systems. Free Radic Biomed. 18,1995: $125-126$

[9]. D.I. Thurnham, (). Antioxidants and prooxidants in malnourished populations. Proceedings of Nutritional Society. 49, 1996 : $173-$ 185

[10]. S.K. Gaby and V.N. Singh, Vitamin C, intake and health; A scientific review(New York, Marcel Dekker,1990) (1033-1044)

[11]. J.H. Weisburger, Nutritional approach to cancer prevention with emphasis on vitamin antioxidants and Carotenoids. American Journal of Nutrition. 53, 1991: 2265-2375

[12]. N. Miller, C. Rice-Evans and M.J. Davies, A novel method for measuring antioxidant capacity and its application to monitoring the antioxidant status in premature neonates. Clinical Science. 84, 1993: 407-412

[13]. M. Cheesbrough,District Laboratory Practice in Tropical Countries. (2 ${ }^{\text {nd }}$ Ed.Part 1. London, Cambridge University Press, 2000)(245-249)

[14]. P. Fossati, L. Prencipe, and G. Berti, (). Use of 3, 5-dichloro-2-hydroxybenzenesulfonic acid/4-aminophenazone chromogenic system in direct enzymic assay of uric acid in serum and urine. Clin. Chem. 26, 1980: 227-231

[15]. J.M.C. Gutteridge and S. Wilkins, Copper-dependent hydroxyl radical damage to ascorbic acid formation of a thiobarbituric acidreactive product. 137 (2)1982: 327-330

[16]. D. Koracevic, G.D. Koracenc and V. Djevic, Method for the measurement of antioxidant activity in human fluids. Journal of Clin. Pathol. 54, 2001: 356-361

[17]. H.T.Malloyand K.A.Evelyn. The determination of bilirubin with the photoelectric colorimeter J. Biol. Chem. 119, 1937: 481-9

[18]. S. A. Buege, J.A. Buege, F.O. O'NeaI and S. D. Aust, The Mechanism of NADPH-Dependent Lipid Peroxidation the Propagation of Lipid Peroxidation J. Biol. Chem. 254, 1978: 5892589

[19]. B.S. Das, J.K. Patnaik, S. Mohanty, S.K. Mishra, D. Mohanty, S.K. Satpathy, and T.K. Bose, (). Plasma antioxidants and lipid peroxidation products in falciparum malaria. Am J Trop Med Hyg. 49 (6) 1993: 720-27

[20]. R. Selvam and S.T. Mathews, Biochemical alterations in Plasmodium vivax infected malarial patients before and after radical treatment. Indian J Malariol. 29, 1992: 103-111

[21]. R. Stocker, N.H. Hunt and M.J. Weidemann, (). Antioxidants in plasma from mice infected with Plasmodium vinckei. Biochem. Biophy. Res Commun. 134, 1986: 152-158

[22]. P. Reyes, P.K. Rathod, D.J. Sanchez, J.E. Mrema, and K.H. Rieckmann, Enzymes of purine and pyrimidine metabolism from the human malaria parasite, Plasmodium falciparum. Mol Biochem. Parasitol 5,1982: 275-290

[23]. W.R. Heath, and F.R. Carbone, Immunology: dangerous liaisons. Nature. 425, 2003:460-461

[24]. L.G. Sanchez-Lozada, T. Nakagawa, D.H. Kang, D.I. Feig, and M. Franco, (). Hormonal and cytokine effects of uric acid. Curr Opin Nephrol Hypertens. 15, 2006:30-33

[25]. Kanellis, J. and Kang, D.H. (). Uric acid as a mediator of endothelial dysfunction, inflammation, and vascular disease. Semin Nephrol. 25, 2005: 39-42 
[26]. J. Kanellis, S. Watanabe, J.H Li, D.H. Kangand P. Li, Uric acid stimulates monocyte chemoattractant protein-1 production in vascular smooth muscle cells via mitogen-activated protein kinase and cyclooxygenase-2. Hypertension. 41, 2003:1287-1293.

[27]. D.H. Kang, T. Nakagawa, L. Feng, S. Watanabe and L. Han, A role for uric acid in the progression of renal disease. J Am Soc. Nephrol. 13, 2002: 2888-2897

[28]. J.O. Akpotuzor, A.E. Udoh, and M.H. Etukudo, High Density Lipoprotein, Total Cholesterol, Albumin, Vitamin C and Total Antioxidant Levels of Children with P. Falciparum Infection in Calabar. Adv.in Med. Dent. Sci. 3 (1), 2009: 4-12

[29]. A.O. Egwunyenga, G. Isamah, and O.P.Nmorsi, Lipid peroxidation and ascorbic acid levels in Nigeria children with acute falciparum malaria.African Journal of Biotechnology. 3 (10), 2004: 560-563

[30]. G. K. Isamah, and S. O. Asagba, The effect of acute Plasmodium falciparum Infection on the levels of malondialdehyde and ascorbic acid on Nigerian Children. Journal of Applied Sciences \& Environmental Management. 7 (2),2003: 59-61

[31]. N.H. Hunt and R. Stocker, Oxidative stress and redox status of malaria infected erythrocytes. Blood Cells. 16, 1990: 499-526

[32]. R.N. Rath, N. Panigrahi, B.K. Das, and P.K. Das, Lipid peroxidation in acute falciparum malaria. Ind. J. Med. Res. 93,1991: 303305

[33]. N.C. Mishra, I. Kabilan and A. Sharma, Oxidative stress and malaria infected erythrocytes. Indian J. Malariol. 31, 1994: 77-87

[34]. O.G. Adeosun, O. T Akanji, B. O. Sunday, A. M. Udoh, and S. J. Bello, Biochemical alteration in Nigerian children with acute falciparum malaria. African Journal of Biotechnology. 6 (7), 2007: 881-885

[35]. K. Becker, L. Tilley, J.L. Vennerstrom, D. Roberts, S. Rogerson and H. Ginsburg, Oxidative stress in malaria parasite-infected erythrocytes: host-parasite interactions. Int J Parasitol. 34 (2), 2004: 163-89 